\title{
European Antibiotic Awareness Day 2017: training the next generation of health care professionals in antibiotic stewardship
}

\author{
Lenneke Schrier $^{1,2}$ (10) Adamos Hadjipanayis ${ }^{1,3,4}$ - Stefano del Torso ${ }^{1,5} \cdot$ Tom Stiris $^{1,6}$ - Marieke Emonts ${ }^{7,8,9}$. \\ Hans Juergen Dornbusch ${ }^{1,10}$
}

Received: 18 October 2017 / Revised: 17 November 2017 / Accepted: 22 November 2017 / Published online: 5 December 2017

(C) The Author(s) 2017. This article is an open access publication

\begin{abstract}
Antimicrobial stewardship (AMS) aims to optimise treatment, minimise the risk of adverse effects and reduce health care costs. In addition, it is recognised as a key component to stop the current spread of antimicrobial resistance in Europe. Educational programmes are particularly important for the successful implementation of AMS. Training should start during medical school, continue during clinical training and be reinforced throughout postgraduate training. National core curricula for paediatric training should include passive and active training of competencies needed for AMS and future paediatricians should be skilled in taking leadership roles in AMS initiatives. Other core members of the paediatric AMS team should also receive training focused on the unique medical needs of the paediatric patient.

Conclusion: Ideally, all communities, hospitals and health regions in Europe should have AMS that serve all patient types, including children. We all have the responsibility to ensure that existing antibiotics remain effective.

\section{What is Known:}

- Antimicrobial stewardship (AMS) is a key component to stop the current spread of antimicrobial resistance

- Educational programmes are particularly important for the successful implementation of AMS

What is New:

- All medical doctors in Europe who will be undertaking significant practice in child health should master the competencies needed to prescribe antibiotics to children rationally as described in the European Academy of Paediatrics (EAP) Curriculum for Common Trunk Training in Paediatrics

- Interdisciplinary approaches of education need to be developed, as all hospitals and health regions in Europe ideally should have AMS programmes that serve all patient types, including children
\end{abstract}

Communicated by Nicole Ritz

Lenneke Schrier

1.schrier@lumc.nl

Adamos Hadjipanayis

adamos@paidiatros.com

Stefano del Torso

deltorso@gmail.com

Tom Stiris

tom.stiris@medisin.uio.no

Marieke Emonts

marieke.emonts@newcastle.ac.uk

Hans Juergen Dornbusch

hansjuergen.dornbusch@medunigraz.at

1 European Academy of Paediatrics, Brussels, Belgium
2 Willem-Alexancer Children's Hospital, Leiden University Medical Center, Leiden, The Netherlands

3 Paediatric Department, Larnaca General Hospital, Larnaca, Cyprus

4 European University Medical School, Nicosia, Cyprus

5 Pediatra di Famiglia, Padua, Italy

6 Department of Neonatal Intensive Care, Oslo University Hospital, Oslo, Norway

7 Institute of Cellular Medicine, Newcastle University, Newcastle upon Tyne, UK

8 Department of Paediatric Immunology and Infectious Diseases, Newcastle upon Tyne, UK

9 Hospital Foundation Trust, Great North Children's Hospital, Newcastle upon Tyne, UK

10 Medical University of Graz, Graz, Austria 
Keywords Antimicrobial resistance $\cdot$ Postdoctoral training $\cdot$ Paediatrics $\cdot$ Antibiotic stewardship $\cdot$ Antibiotics

\author{
Abbreviations \\ AMR Antimicrobial resistance \\ AMS Antimicrobial stewardship \\ CRP C-reactive protein \\ EAP European Academy of Paediatrics \\ ESPID European Society for Paediatric Infectious Diseases
}

\section{Introduction}

Antimicrobial resistance (AMR) poses a serious and increasing threat to public health as infections caused by bacteria that are resistant to antimicrobials lead to approximately 25,000 deaths in the European Union every year [7]. In children, a significant increase in the prevalence of multidrug-resistant bacterial infections has occurred during the past couple of decades [5]. Over the last few years, there have been significant shortages in the development and availability of new antibiotics, and the number of targeted studies on antibiotics in children remains strikingly low [23]. Therefore, the implementation of strategies to preserve the efficacy of existing antibiotics is an urgent public health priority, both in hospital and community settings.

Antimicrobial stewardship (AMS) aims to appropriately and safely prescribe antibiotics to patients, while reducing unnecessary or suboptimal use of antibiotics, thus maximising outcomes for the patient [2]. AMS is recognised as a key component to stop the current spread of AMR in Europe. AMS programmes have the ultimate goal of minimising selective pressure on the emergence of drug-resistant strains. Successful AMS programmes are characterised by the ability to break down activities into specific interventions that can be more easily implemented, monitored and evaluated [3]. Successfully implemented AMS programmes have a significant impact on reducing antimicrobial use in paediatric patients, costs and prescribing errors without negative impacts on patient safety, and actually resulting in improved patient outcomes $[13,23,24]$. The identification of paediatric conditions with both frequent and variable antimicrobial use could guide the prioritisation of high-impact targets for AMS interventions [10]. Paediatric AMS should not be limited to the hospital setting and collaboration among hospital and outpatient health care facilities is of paramount importance [12, 14]. Indeed, AMS can be effective in reducing antibiotic misuse in community settings $[9,11$, 26], and potential strategies to promote these programmes in community-based settings have been published [14].

\section{Training in antimicrobial stewardship}

As active participation of clinical professionals at all levels of care is required for AMS to succeed, educational programmes are particularly important for the successful implementation of AMS [25]. Education of health care providers on appropriate antibiotic prescribing has been shown to enhance other antimicrobial stewardship interventions [22]. Therefore, the European Academy of Paediatrics (EAP) Curriculum for Common Trunk Training in Paediatrics [6] includes specific knowledge and skills related to AMR and the European Society for Paediatric Infectious Diseases (ESPID) and other national (paediatric) infectious diseases groups run specific courses on AMR (Table 2). The syllabus for Core Training sets out a European road map for common paediatric training. It is intended as a guide for national paediatric societies to help them understand the principles of core training. The EAP recognises that in many countries in Europe, training does not conform to these recommendations, but as such differences gradually diminish, quality, content and assessment of training, including training related to the principles AMR, will become more uniform across Europe. At the same time, it is recognised that differences in regional epidemiology and healthcare infrastructure require a tailor-made approach, also for AMS. Recently, recommendations were published for key points to be included in clinical curricula, in order to develop the necessary skills to participate in AMS [20,22]. These are summarised in Table 1. It is important that assimilation of knowledge about AMS starts already during medical school, is continued during clinical training and is reinforced throughout postgraduate training. Passive educational techniques like large and small group presentations are modestly effective for increasing knowledge. However, interactive or dynamic techniques influence prescribing behaviour. This includes education associated with specific episodes of patient care. Interactive small group sessions, e-learning, educational outreach, periodic retrospective audit and feedback and one-on-one patient-directed education have been shown to be moderately to highly effective in optimising antibiotic use and patient outcomes [22]. In particular, internet-based education shows encouraging results $[15,17,18]$. The advantages of e-learning include improved access to education in rural or low-resource areas and the potential to develop an interactive platform. Several examples of e-learning tools are listed in Table 2. In addition, AMS was recently taught during the EAP MasterCourse 2017 and is included in the upcoming European Academy of Paediatric Societies congress in Paris, 2018. In addition, AMS is always covered during the annual residential ESPID-Oxford Course.

Although many of the overarching principles of AMS apply to children and adults alike, many factors related to paediatric AMS are unique to children. Children have high rates of infection and frequently present with non-specific symptoms adding to diagnostic uncertainty. Patterns of infection 
Table 1 Key points to include in clinical AMS curricula [adapted from [20, 22]

Learning objective(s)

The participant learns about how AMS contributes to accurate and safer prescribing of antimicrobials, resulting in improved clinical outcomes

Effect of AMS initiatives on bacterial resistance

Diagnosis of infection

Principles of infection management in children

Prescribing of antibiotics

Guidelines for diagnosis and management of most frequent infections in children

Prevention of infection

Communication skills
The participant learns about antibiotic resistance mechanisms, including causes and extent

The participant learns how AMS reduces the spread of antimicrobial resistance

The participant learns how to accurately interpret laboratory reports in order to make clinical treatment decisions in neonates, infants, children and adolescents;

The participant learns how to diagnose an infection in a standardised manner;

The participant learns how to make informed treatment decisions early in the course of disease in order to positively influence treatment outcomes;

The participant learns how to de-escalate antibiotic use in order to more effectively treat an infection while limiting exposure to broad-spectrum antimicrobials

The participant learns the basics needed to prescribe antibiotics for infections caused by susceptible and resistant organisms

The participant learns about the importance of preventive measures to limit the development of antimicrobial resistance

The participant learns how to apply communication techniques to talk with patients and families about prudent antibiotic use
Topics

Effective treatment

Clinical outcomes (mortality and morbidity)

Reduction of side effects

Population specific approaches

Epidemiology (global)

Genetics and mechanisms

Relationship to antibiotic use

Discussion of AMS initiatives like prospective audit with feedback and formulary restriction

Proper use and interpretation of bacterial Gram stain/culture, rapid and point-of-care tests, serology, and biomarkers of infection

Establishment of standardised diagnostic criteria for specific infections

Promptly identify patients who require antibiotics

Timely and appropriate initiation of antibiotics

Obtain cultures before starting antibiotics

Do not give antibiotics with overlapping activity or combinations not supported by evidence or guidelines

Determine and verify antibiotic allergies

Consider local antibiotic susceptibility

Specify expected duration of therapy based on evidence and national and hospital guidelines

Ensure appropriate administration (intravenous versus oral)

Give antibiotics at the right dose and interval

Stop or de-escalate therapy promptly based on the culture and sensitivity results or establishment of an alternative diagnosis

Reconcile and adjust antibiotics at all transitions and changes in patient's condition

Monitor for toxicity reliably and adjust agent and dose promptly

Pharmacokinetics and mechanism of action of different classes of antibiotics ('bug-drug' coverage)

Pharmacology and adverse effects, including risk of C. difficile infection

Principles of empirical versus directed therapy

Drug purchasing and dispensing costs

Specific instruction for these common infections using principles outlined above

Use of national and local guidelines and public health guidance

Hand hygiene

Prudent use of catheters and devices

Principles and duration of surgical prophylaxis 
Table 2 Available e-learning tools

AMS (general)

Collection of AMS online courses made by the European Centre for Disease Prevention and Control: https://ecdc.europa.eu/en/publications-data/directory-guidance-prevention-and-control/training-antimicrobial-stewardship Massive Open Online Course on Antimicrobial Stewardship (University of Dundee, UK): https://www.futurelearn.com/courses/antimicrobial-stewardship Antimicrobial Stewardship Online CME Courses (Stanford University School of Medicine, USA): https://med.stanford.edu/cme/learning-opportunities/antimicrobialstewardship.html

Paediatric AMS

European Society for Paediatric Infectious Diseases (ESPID) online course on paediatric AMS:

http://www.espid.org/content.aspx?Page=ESPID\%20Online\%20Antibiotic\%20Management\%20Course

and resistance vary significantly by age, thus age-specific antibiotic panels for antibiograms should preferably be used to guide antibiotic choices for selected infections. Children are more prone to infection with resistant organisms due to a future lifetime of antibiotic exposure. In addition, considerations related to age-appropriate dosing and formulations pose challenges to the prescription of antibiotics in children [4]. Children may respond differently to antibiotics compared to adults. Finally, immunisation initiatives should be included in AMS programmes as a preventive strategy in both in- and outpatient paediatric settings, in order to decrease the likelihood of serious illness and to decrease AMR. Therefore, other core members of the paediatric AMS team, like the microbiologist, paediatric infectious disease specialist and clinical pharmacist, should receive training focused on the unique medical needs of the paediatric patient $[16,19]$. In addition, future paediatricians should be skilled in taking leadership roles in AMS initiatives and develop practical solutions rooted in the general principles of AMS, as it is important to further expand AMS activities from the hospital to paediatric offices and communities [14].

\section{Keep antibiotics working}

Limiting the further spread of AMR is one of EAP's child health priorities for 2017-2018. We will work together with the European Centre for Disease Prevention and Control to raise awareness about the relevance and benefits of costeffective AMS policies [21] and training in child health and to advocate for sustained implementation of these across Europe. Europe would greatly benefit from a uniform adoption of AMR and infection prevention best practice across countries. This includes harmonisation and simplification of the various treatment guidelines that currently exist across Europe. Moreover, there is an urgent need to conduct research on new antibiotic (classes) for critical multi-drug-resistant pathogens and on the effects of AMS programmes in lowincome countries in which emerging resistance are particularly alarming [1]. In addition, limited access to high-quality antibiotics is particularly of concern in these countries. Continuous education and training of health care professionals on appropriate antibiotic use is crucial. We therefore welcome the new European Action Plan, which was launched this summer. The European Commission intends, among others, to develop training programmes on AMR for health professionals through the ECDC and the EU health programme [8].

\section{Conclusion}

Prevention of AMR needs rigorous actions in the community, at practice, ward, institutional, national and international levels. Best practices should be applied cross-border and healthcare institutions and communities should collaborate regionally and internationally, in order to fight AMR successfully. Effective Europe-wide implementation and sustained use of cost-effective antimicrobial policies can consequently lead to improved safety and quality of care while contributing to more sustainable healthcare. Ideally, all communities, hospitals and health regions in Europe should have AMS programmes that serve all patient types, including children. This includes adult academic and community hospitals and outpatient care centres that primarily care for children. Therefore, the EAP is interested to discuss interdisciplinary approaches of education with other stakeholders.

Prudent use of antibiotics is very important to address the global challenges posed by AMR. We all have the responsibility to ensure that existing antibiotics remain effective.

Authors' contributions Lenneke Schrier initiated the EAP statement (together with HJD), wrote the draft and agrees on its final wording. Adamos Hadjipanayis commented on the draft and agrees on its final wording. Stefano del Torso commented on the draft and agrees on its final wording. Tom Stiris commented on the draft and agrees on its final wording. Marieke Emonts commented on the draft and agrees on its final wording. Hans Juergen Dornbusch initiated the EAP statement (together with LS), commented on the draft and agrees on its final wording.

\section{Compliance with ethical standards}

Conflict of interest The authors declare that they have no conflict of interest.

Ethical approval This article does not contain any studies with human participants or animals performed by any of the authors and therefore neither ethical approval nor informed consent applies. 
Open Access This article is distributed under the terms of the Creative Commons Attribution 4.0 International License (http:// creativecommons.org/licenses/by/4.0/), which permits unrestricted use, distribution, and reproduction in any medium, provided you give appropriate credit to the original author(s) and the source, provide a link to the Creative Commons license, and indicate if changes were made.

\section{References}

1. Araujo da Silva AR, Albemaz de Alemeida Dias DC, Marques AF, et al. (2017) The role of antimicrobial stewardship programmes in children: a systematic review. J Hosp Infect pii S0195-6701(17): 30447-30444. https://doi.org/10.1016/j.jhin.2017.08.003

2. Baur D, Gladstone BP, Burkert F, Carrara E, Foschi F, Dobele S, Tacconelli E (2017) Effect of antibiotic stewardship on the incidence of infection and colonisation with antibiotic-resistant bacteria and Clostridium difficile infection: a systematic review and metaanalysis. Lancet Infect Dis 17(9):990-1001. https://doi.org/10. 1016/S1473-3099(17)30325-0

3. Bielicki JB, Lundin RL, Patel S, Paulus S (2015) Antimicrobial stewardship for neonates and children: a global approach. Pediatr Infect Dis J 34(3):311-313. https://doi. org/10.1097/INF.0000000000000621

4. Downes KJ, Hahn A, Wiles J, Courter JD, Vinks AA (2014) Dose optimisation of antibiotics in children: application of pharmacokinetics/pharmacodynamics in paediatrics. Int $\mathrm{J}$ Antimicrob Agents 43(3):223-230. https://doi.org/10.1016/j. ijantimicag.2013.11.006. Epub 2013 Dec 17

5. Elliot SP (2008) Antimicrobial-resistant pathogens: an emerging pediatric threat. Adv Pediatr Infect Dis 55:329-348

6. European Academy of Paediatrics Curriculum for Common Trunk Training in Paediatrics, agreed by the general assembly in Brussels, $6^{\text {th }}$ December 2014. URL: http://eapaediatrics.eu/wp-content/ uploads/2015/12/Agreed-Common-trunk-curriculum-trainingLAST1.pdf

7. European Centre for Disease Prevention and Control/European Medicines Agency (2009) The bacterial challenge: time to react. Joint Technical Report, EMEA doc. ref. EMEA/576176/2009

8. European Commission (2017) A Europe One Health Action Plan against Antimicrobial Resistance. URL: http://ec.europa.eu/health/ amr/action eu en. Accessed 16 Nov 2017

9. Finkelstein JA, Huan SS, Kleinman K et al (2008) Impact of a 16-community trial to promote judicious antibiotic use in Massachusetts. Pediatrics 121(1):e15-e23. https://doi.org/10. 1542/peds.2007-0819

10. Gerber JS, Kronman MP, Ross RK et al (2013) Identifying targets for antimicrobial stewardship in children's hospitals. Infect Control Hosp Epidemiol 34(12):1252-1258

11. Gerber JS, Prasad PA, Fiks AG et al (2013) Effect of an outpatient antimicrobial stewardship intervention on broad-spectrum antibiotic prescribing by primary care pediatricians: a randomized trial. JAMA 309:2345-2352

12. Goldman JL, Newland JG (2015) New horizons for pediatric antibiotic stewardship. Infect Dis Clin N Am 29(3):503-511. https://doi.org/10.1016/j.idc.2015.05.003

13. Hersh AL, De Lurgio SA, Thurm C, Lee BR, Weissman SJ, Courter JD, Brogan TV, Shah SS, Kronman MP, Gerber JS, Newland JG
(2015) Antimicrobial stewardship programs in freestanding children's hospitals. Pediatrics 135(1):33-39. https://doi.org/10.1542/ peds.2014-2579

14. Hyun DY, Hersh AL, Namtu K, Palazzi DL, Maples HD, Newland JG, Saiman L (2013) Antimicrobial stewardship in pediatrics: how every pediatrician can be a steward. JAMA Pediatr 167(9):859 866. https://doi.org/10.1001/jamapediatrics.2013.2241

15. Little P, Stuart B, Francis N, Douglas E, Tonkin-Crine S, Anthierens S, Cals JW, Melbye H, Santer M, Moore M, Coenen S, Butler C, Hood K, Kelly M, Godycki-Cwirko M, Mierzecki A, Torres A, Llor C, Davies M, Mullee M, O'Reilly G, van der Velden A, Geraghty AW, Goossens H, Verheij T, Yardley L, GRACE consortium (2013) Effects of internet-based training on antibiotic prescribing rates for acute respiratory-tract infections: a multinational, cluster, randomised, factorial, controlled trial. Lancet 382(9899):11751182. https://doi.org/10.1016/S0140-6736(13)60994-0

16. Magsarili HK, Girotto JE, Bennett NJ, Nicolau DP (2015) Making a case for pediatric antimicrobial stewardship programs. Pharmacotherapy 35(11):1026-1036. https://doi. org/10.1002/phar.1647

17. McNulty CA (2012) European Antibiotic Awareness Day 2012: general practitioners encouraged to TARGET antibiotics through guidance, education and tools. J Antimicrob Chemother 67(11): 2543-2546. https://doi.org/10.1093/jac/dks358

18. Nathwani D, Sneddon J, Malcolm W, Wiuff C, Patton A, Hurding S, Eastaway A, Seaton RA, Watson E, Gillies E, Davey P, Bennie M, Scottish Antimicrobial Prescribing Group (2011) Scottish Antimicrobial Prescribing Group (SAPG): development and impact of the Scottish National Antimicrobial Stewardship Programme. Int J Antimicrob Agents 38(1):16-26. https://doi.org/10.1016/j. ijantimicag.2011.02.005

19. Nichols K, Stoffella S, Meyers R, Girotto J, Advocacy Committee for the Pediatric Pharmacy Advocacy Group (2017) Pediatric antimicrobial stewardship programs. J Pediatr Pharmacol Ther 22(1): 77-80. https://doi.org/10.5863/1551-6776-22.1.77

20. O'Donnell LA, Guarascio AJ (2017) The intersection of antimicrobial stewardship and microbiology: educating the next generation of health care professionals. FEMS Microbiol Lett 364:fnw281

21. Oberjé EJM, Tanke MAC, Jeurissen PPT (2017) Antimicrobial stewardship initiatives throughout Europe: proven value for money. Infect Dis Rep 9(1):6800. https://doi.org/10.4081/idr.2017.6800

22. Ohl CA, Luther VP (2014) Health care provider education as a tool to enhance antibiotic stewardship practices. Infect Dis Clin N Am 28(2):177-193. https://doi.org/10.1016/j.idc.2014.02.001

23. Principi N, Esposito S (2016) Antimicrobial stewardship in paediatrics. BMC Infect Dis 16(1):424. https://doi.org/10.1186/s12879016-1772-z

24. Smith MJ, Gerver JS, Hersh AL (2015) Inpatient antimicrobial stewardship in pediatrics: a systematic review. J Pediatr Infect Dis Soc 4(4):e127-e135. https://doi.org/10.1093/jpids/piu141

25. Society for Healthcare Epidemiology of America, Infectious Diseases Society of America, Pediatric Infectious Diseases Society (2012) Policy statement on antimicrobial stewardship by the SHEA, IDSA and PIDS. Infect Control Hosp Epidemiol 33: 322-327

26. Swedres Swarm (2014) Consumption of antibiotics and occurrence of antibiotic resistance in Sweden. URL: http://www.sva.se/ globalassets/redesign2011/pdf/om_sva/publikationer/swedres svarm2015.pdf. 